\title{
Alignment-Dependent Atomic Model for Electron Transfer in Ion-Molecule Collisions
}

\section{D. Lin ${ }^{*}$ and Ingrid Reiser}

J. R. Macdonald Laboratory, Department of Physics, Kansas State University, Manhattan, KS 66506 U.S.A.

*Author to whom correspondence should be addressed. E-mail: cdlin@phys.ksu.edu

Received: 23 October 2001/ Accepted: 19 November 2001/ Published: 28 March 2002

\begin{abstract}
The alignment-dependent atomic model for treating electron transfer processes in ion-molecule collisions [Shingal and Lin, Phys. Rev. 1989, A40, 1302] is reviewed. This model has been applied to collisions between $\mathrm{H}^{+}$and $\mathrm{He}^{2+}$ with $\mathrm{H}_{2}$, and recently also to collisions between $\mathrm{He}^{2+}$ and $\mathrm{Ar}^{2+}$ ions with $\mathrm{H}_{2}{ }^{+}$, where alignment effect is being explored experimentally.
\end{abstract}

Keywords: ion-molecule collisions; close-coupling method; alignment effect; ion-ion collisions.

\section{Introduction}

Electron captures from atoms or molecules in collisions with ions in the $\mathrm{keV}$ to $\mathrm{MeV}$ energy region have been extensively studied over the last few decades. For simple atomic targets with one or two active electrons the close-coupling method, based either on atomic orbitals (AO) [1] or molecular orbitals (MO) [2], has been successfully applied to obtain accurate cross sections for ion-atom collisions. The close-coupling method can in principle be generalized to treat ion-molecule collisions $[3,4]$. However, due to the computational complexity, few calculations have been carried out. To perform ion-molecule collisions within the molecular orbital expansion method, on the one hand, one needs to generate accurate two-center or many-center molecular wavefunctions which makes the computation of rearrangement matrix elements very tedious. On the other hand, one needs to perform calculations for different molecular alignment. Since most experiments do not explore the alignment of the molecule in ionmolecule collisions, such effect is averaged over in order to compare with experiments. To bypass such complications, in the literature many theoretical calculations for ion-molecule collisions make the 
drastic approximation by treating molecules as spherical, as in an atom, with an effective charge fitted to reproduce the correct ionization energy. Such simplification neglects the nonisotropic electronic density distribution of a molecule and the dependence of electron capture cross sections on the alignment of the molecule is completely neglected.

There have been very few experiments investigating the dependence of electron capture cross sections on the alignment of the molecules. Consider diatomic molecular targets only, for ion energies of the order of $\mathrm{keV} / \mathrm{amu}$ and higher, the rotational and vibrational periods are much longer than the collision time such that electron capture occurs from molecules fixed in space. Experimentally the difficulty lies in preparing aligned molecules. However, the alignment of the molecule can be determined if the resulting molecular ion is unstable and breaks into charged particles. For single electron capture from a neutral diatomic molecule, the resulting molecular ion is stable for the dominant channels. Thus the alignment effect from neutral molecules have been investigated for double ionization and transfer excitation processes only, for example, from $\mathrm{D}_{2}$ molecules by $\mathrm{F}^{8+}$ ions [5]. These experiments are more difficult to interpret theoretically due to the presence of many dissociation channels and the fact that two-electron transitions are much more difficult to treat theoretically. Nevertheless, alignment effect has been observed in these experiments and there are theoretical models aimed at interpreting these observations [6].

From the theoretical viewpoint, the most elementary ion-molecule collision system is a one-electron molecular target, such as $\mathrm{H}_{2}{ }^{+}$. The advantage of such a target is that after electron capture, the molecule breakups into two charged particles which can be measured directly to infer the alignment of the molecular ion. The resulting electron capture cross sections can be used to test ab initio calculations directly, or to test the validity of the simpler theoretical models. However, collision experiments between two positive ions are very difficult [7]. Recently, great progress has been made by experimentalists and total electron capture cross sections in collisions between atomic ions and $\mathrm{H}_{2}^{+}$have already been reported [8]. The next step is to detect both fragments in coincidence to extract the alignment effect.

In this contribution our aim is to investigate effect of the alignment of molecules in electron capture process in ion-molecule collisions. While it may be possible to perform the standard ion-molecule collisions using the MO approach, we will instead adopt the simple alignment-dependent atomic model previously proposed by Shingal and Lin[9 ]. The model is based on amplitudes calculated from ionatom collisions but the effect of the two atomic centers in a molecule and the alignment of the molecule with respect to the ion beam is accounted for. This simple model makes the study of the alignment effect more transparent and the calculation can be carried out readily. Applications of this model to collisions with $\mathrm{H}_{2}$ and $\mathrm{H}_{2}^{+}$targets will be addressed.

In the model of Shingal and Lin the target wavefunction of the molecule is written as a simple linear combination of atomic orbitals from different centers. The electron capture amplitude for ion-molecule collisions is expressed as the linear superposition of ion-atom collision amplitudes between the projectile ion and the different atomic centers. In such a model, only the ion-atom collision amplitude has to be calculated which can be readily obtained from standard close-coupling codes. The effect of the molecular alignment is taken care of by accounting for the relative phases for scattering from the different atomic centers. In Section II, the theoretical model is discussed, together with a brief description of the experimental method for doing ion- $\mathrm{H}_{2}{ }^{+}$collisions. Typical results from $\mathrm{H}^{+}$and $\mathrm{He}^{2+}$ collisions 
with $\mathrm{H}_{2}$ and newer data from $\mathrm{He}^{2+}$ and $\mathrm{Ar}^{2+}$ collisions with $\mathrm{H}_{2}{ }^{+}$are presented in Section III. A short summary is given in Section IV.

\section{The Theoretical Model and Experimental Method for ion- $\mathrm{H}_{2}{ }^{+}$Collisions \\ (a) Theoretical Model}

Consider the collision between a projectile with a diatomic molecule with the geometry shown in Fig. 1. The origin of the coordinate system is taken at the center $\mathrm{C}$ of the molecule $\mathrm{AB}$. We will

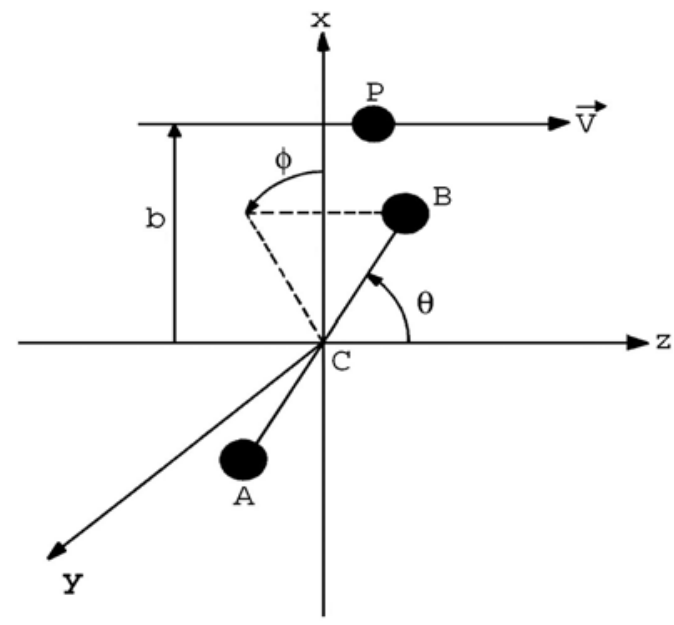

Fig.1. Coordinate system in the laboratory frame for ion- $\mathrm{H}_{2}$ collisions.

assume that there is only one active electron which has coordinates $\mathbf{r}_{\mathbf{A}}$ and $\mathbf{r}_{\mathbf{B}}$ with respect to A and B, respectively, and $\mathbf{r}_{\mathbf{P}}$ with respect to the projectile. In the perturbation approximation, the amplitude $a_{\mathrm{fi}}(\mathrm{b})$ for transition from the initial state $\mid \mathrm{i}>$ to final state $|\mathrm{f}\rangle$ is obtained from

$$
\mathrm{a}_{\mathrm{fi}}=\left\langle\Psi_{\mathrm{f}}\left|\mathrm{V}_{\mathrm{P}}\right| \Psi_{\mathrm{i}}\right\rangle
$$

where $V_{P}$ is the electron-projectile interaction. In (1), the time-dependent initial state wavefunction is given by

$$
\Psi_{i}=\psi_{i}(\vec{r}) \exp \left(-i \varepsilon_{i} t\right)
$$

and the final state time-dependent wavefunction is given by

$$
\Psi_{f}=\psi_{f}\left(\vec{r}_{p}\right) \exp \left(i \vec{v} \cdot \vec{r}-i v^{2} t / 2-i \varepsilon_{f} t\right)
$$

where $\psi_{\mathrm{f}}$ is the electronic wavefunction centered at the projectile. Since the projectile is moving at a velocity $\mathrm{v}$ with respect to the origin of the target molecule, a plane-wave electron translational factor is included in the time-dependent wavefunction. Atomic units are used throughout this paper unless otherwise noted. If one writes the initial state electronic wavefunction for the diatomic molecule as

$$
\Psi_{i}=\left[\phi\left(\vec{r}_{A}\right)+\phi\left(\vec{r}_{B}\right)\right] / \sqrt{2},
$$


then the scattering amplitudes can be expressed explicitly as

$$
\begin{aligned}
& a_{f i}=\frac{1}{\sqrt{2}}\left[\int d \vec{r} d t V_{P} \psi_{f}\left(\vec{r}_{P}\right) \phi\left(\vec{r}_{A}\right)\right. \\
& \times \exp \left(-i \vec{v} \cdot \vec{r}+i \omega t+i v^{2} t / 2\right) \\
&+\int d \vec{r} d t V_{P} \psi_{f}\left(\vec{r}_{P}\right) \phi\left(\vec{r}_{B}\right) \\
&\left.\times \exp \left(-i \vec{v} \cdot \vec{r}+i \omega t+i v^{2} t / 2\right)\right],
\end{aligned}
$$

where $\omega=\varepsilon_{\mathrm{f}}-\varepsilon_{\mathrm{i}}$. The quantities above are defined with respect to the origin at $\mathrm{C}$. We note that the first integral is identical to the electron capture amplitude between the projectile with atom A except for the constant phase factor, and the second integral is similar to the amplitude for capture from atom B, except for a phase factor. To find the phase factor, assume that the time integration with respect to $\mathrm{C}$ is from $-\mathrm{T}$ to $+\mathrm{T}$, where $\mathrm{t}=0$ is taken to be when the projectile is at the distance of closest approach. By shifting the integration from $\mathrm{d} \mathbf{r}$ to $\mathrm{d} \mathbf{r}_{\mathbf{A}}$, the first integral can be written as

$$
\begin{aligned}
\int_{-T}^{\mathrm{T}} \mathrm{dt} \int \mathrm{dr}_{\mathrm{A}} & \mathrm{V}_{\mathrm{P}} \psi_{\mathrm{f}}\left(\overrightarrow{\mathrm{r}}_{\mathrm{P}}\right) \phi\left(\overrightarrow{\mathrm{r}}_{\mathrm{A}}\right) \\
& \times \exp \left[-\mathrm{i} \overrightarrow{\mathrm{v}} \cdot\left(\overrightarrow{\mathrm{r}}_{\mathrm{A}}-\vec{\rho} / 2\right)+\mathrm{i}\left(\omega+\mathrm{v}^{2} / 2\right) \mathrm{t}\right] .
\end{aligned}
$$

where $\vec{\rho}$ is the internuclear distance vector. We next transform the time integration so that $\mathrm{t}^{\prime}=0$ when the projectile is at the distance of closest approach with respect to center A. The time $\tau$ for the wavefront of the projectile to travel from A to $C$ is $\tau=(\rho \cos \theta) /(2 \mathrm{v})$ where $\theta$ is the angle of the molecular axis with respect to the beam direction. Such a shift results in an additional phase factor $\exp [\operatorname{iv}(\rho / 2)$ $\left.\cos \theta-i\left(\omega+v^{2} / 2\right) \tau\right]$. A similar procedure can be applied to the second integral in (5). Retaining only the relative phase, the electron capture amplitude can be expressed as

$$
a_{f i}=\frac{1}{\sqrt{2}}\left\{a\left(\vec{b}_{A}\right)+a\left(\vec{b}_{B}\right) \exp [-i \rho \cos \theta(v / 2-\omega / v)]\right\}
$$

where $\mathbf{b}_{\mathbf{A}}$ and $\mathbf{b}_{\mathbf{B}}$ are the impact parameters with respect to atom $\mathrm{A}$ and atom $\mathrm{B}$, respectively. Thus equation (7) expresses the electron capture amplitudes in ion-molecule collisions in terms of electron capture amplitudes in ion-atom collisions.

Equation (7) was derived using the perturbation theory. The probability for electron capture probability is obtained from

$$
\mathrm{P}(\mathrm{b})=\left|\mathrm{a}_{\mathrm{fi}}\right|^{2}
$$

One would like to extend the present simple model beyond the perturbation regime. Thus one would replace a(b), intsead of being obtained from the perturbation theory, but obtained from the closecoupling calculations. Under such an extension, the probability (8a) can become greater than unity. In such a situation we can perform an artificial "unitarization" by replacing $\mathrm{P}(\mathrm{b})$ by

$$
\mathrm{P}_{\mathrm{u}}(\mathrm{b})=\sin ^{2} \sqrt{\mathrm{P}(\mathrm{b})} \text {. }
$$


To obtain alignment-dependent electron capture cross section, we need to integrate over the impact parameter plane for each molecular alignment angle. Choose z-axis to be along the beam direction, the impact parameter plane is defined to be the xy-plane with

$$
\mathrm{b}=(\mathrm{b} \cos \alpha, \mathrm{b} \sin \alpha, 0)
$$

(see Fig.2). Let $\theta$ and $\phi$ be the azimuthal angles of the molecule, then the position of atom

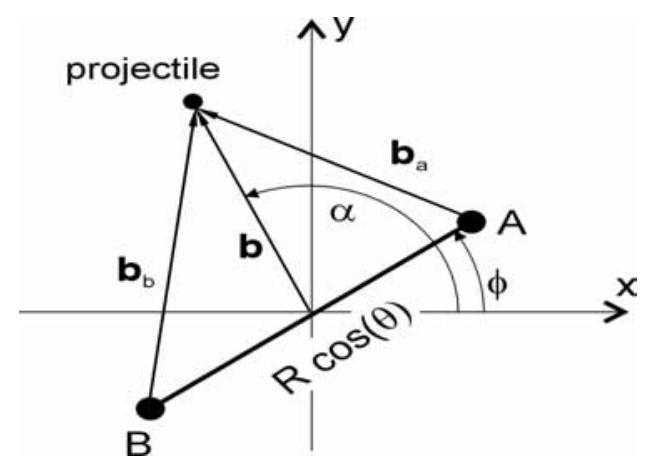

Fig.2. The impact parameter plane in an ion-molecule collision. The projectile is moving out of the plane.

$\mathrm{A}$ is given by

$$
\vec{R}_{A}=\left(x_{A}, y_{A}, z_{A}\right)=-\frac{\rho}{2}(\sin \theta \cos \phi, \sin \theta \sin \phi, \cos \theta)
$$

and $\vec{R}_{B}=-\vec{R}_{A}$. This allows us to calculate $\mathrm{b}_{\mathrm{A}}$ and $\mathrm{b}_{\mathrm{B}}$, i.e., the impact parameters needed for atoms $\mathrm{A}$ and $\mathrm{B}$, respectively,

$$
\begin{aligned}
& b_{A}^{2}=\left(b \cos \alpha-x_{A}\right)^{2}+\left(b \sin \alpha-y_{A}\right)^{2}, \\
& b_{\mathrm{B}}^{2}=\left(\mathrm{b} \cos \alpha-\mathrm{x}_{\mathrm{A}}\right)^{2}+\left(\mathrm{b} \sin \alpha-\mathrm{y}_{\mathrm{A}}\right)^{2} .
\end{aligned}
$$

For each aligned molecule, the probability at a given $b$ is obtained from

$$
\mathrm{P}^{\prime}(\mathrm{b})=\int_{0}^{2 \pi} \mathrm{P}(\mathrm{b}, \alpha) \mathrm{d} \alpha
$$

where $\mathrm{P}(\mathrm{b}, \alpha)$ is obtained from $(8 \mathrm{a})$ or its unitarized version $(8 b)$. The cross section for electron capture from an aligned molecule is then obtained from

$$
\sigma(\theta, \phi)=\int_{0}^{\infty} b P^{\prime}(b) d b
$$

and electron capture cross section averaged over all the alignments of the molecule is given by

$$
\bar{\sigma}=\frac{1}{4 \pi} \int \sigma(\theta, \phi) \mathrm{d} \Omega
$$


(b) Crossed-Beam experiments between $\mathrm{H}_{2}^{+}$and atomic ions.

Experiments to determine absolute total cross sections for the reactions

$$
\begin{aligned}
& \mathrm{H}_{2}^{+}+\mathrm{He}^{2+} \rightarrow 2 \mathrm{H}^{+}+\mathrm{He}^{+} \\
& \mathrm{H}_{2}{ }^{+}+\mathrm{Ar}^{2+} \rightarrow 2 \mathrm{H}^{+}+\mathrm{Ar}^{+}
\end{aligned}
$$

have been carried out using the Giessen ion-ion collision apparatus [8]. Both the $\mathrm{H}_{2}{ }^{+}$and the doubly charged projectile ions are produced in ECR ion sources. This results in $\mathrm{H}_{2}{ }^{+}$molecular ions which are vibrationally excited. Assuming that the ions are created from the $\mathrm{H}_{2}$ source gas through an electronic Franck-Condon transition, the vibrational state distribution peaks at $v=2$.

The ion beams are charge-state analyzed by magnets and further charge-state cleaned right before entering the collision region. In the collision region, which is held at pressures below $10^{-10}$ torr to minimize rest gas collisions, the two ion beams intersect each other at an angle of $\beta=17.5^{\circ}$. After the collision, reaction products are separated from the parent ion beams by electrostatic analyzers. The collision products are detected with single-particle-detectors. The true ion-ion collision products are identified through a time-coincidence setup between the charge-changed projectile and one molecular fragment. The total cross section is obtained from the measured true-event rate $\mathrm{R}$ through

$$
\sigma=\frac{\mathrm{R}}{\varepsilon_{1} \varepsilon_{2}} \frac{\mathrm{v}_{1} \mathrm{v}_{2} \sin \beta}{\mathrm{v}_{\text {rel }}} \frac{\mathrm{q}_{1} \mathrm{q}_{2}}{\mathrm{I}_{1} \mathrm{I}_{2}} \mathrm{~F}
$$

where $\varepsilon_{1}$ and $\varepsilon_{2}$ are the efficiencies of the two detectors. In determining the efficiency of the molecular fragment detector, the probability of detecting at least one out of the two fragments is taken into account. $\mathrm{v}_{1}\left(\mathrm{v}_{2}\right)$ are velocities, $\mathrm{q}_{1}\left(\mathrm{q}_{2}\right)$ are charge states of the two ion beams. The beam currents $\mathrm{I}_{1}$ and $\mathrm{I}_{2}$ and the form factor $\mathrm{F}$ are measured at intervals during each measurement, depending on beam stability.

In order to be able to carry out experiments to determine alignment-dependent cross sections, the positions of both molecular fragment have to be detected simultaneously in order to reveal the molecular axis alignment at the time of collision. This can be done using multi-hit capable position-sensitive detectors, provided that the fragments are spread over a time interval which is larger than the pulsepair resolution of the detector. Such experiments are under way at the KSU ion-ion-collision facility.

\section{Results and Discussion}

$\mathrm{H}^{+}$and $\mathrm{He}^{++}$collisions with $\mathrm{H}_{2}$

Experimental total charge transfer cross sections with a $\mathrm{H}_{2}$ target have been determined with many atomic ions over a broad range of energies. In this subsection we illustrate the validity and the limitation of the model of Shingal and Lin for the $\mathrm{H}_{2}$ target. To compare with experiments, the two electrons in $\mathrm{H}_{2}$ are treated as independent. In the independent electron model the probability for electron capture $\mathrm{P}=\mathrm{P}(\mathrm{b}, \alpha)$ in Eq. (12) is replaced by $2 \mathrm{P}(1-\mathrm{P})$ before integrating over $\alpha$ and $\mathrm{b}$.

In Fig. 3 the total charge transfer cross sections for $\mathrm{H}^{+}+\mathrm{H}_{2}$ collisions for proton energies from 1 to $300 \mathrm{keV}$ are shown. The experimental data are from the compilation of Tawara et al [10 ]. For energies 
below $20 \mathrm{keV}$, the close-coupling calculations of Kimura [3] are shown. Note that the simple atomic model described in Section II gives results that are in good agreement with the experimental data [10 ] over a broad energy range. The larger discrepancy at lower energies is mostly due to the failure of the independent electron model and the possible limitation of the unitarization. In obtaining the results in Fig. 3, each atom in $\mathrm{H}_{2}$ is treated as an atomic hydrogen with effective charge 1.09. For $\mathrm{H}^{+}+\mathrm{H}$ collisions in the covered collision energy regime, a two-state atomic orbital expansion including the 1s orbital on each center is known to be adequate. Thus the results in Fig. 3 were obtained with the twostate AO expansion approximation. The theory shows a larger discrepancy at energies near and above $200 \mathrm{keV}$. This can be explained by the fact that it is known that the two-state model tends to overestimate electron capture cross sections in $\mathrm{H}^{+}+\mathrm{H}$ collisions at higher energies.

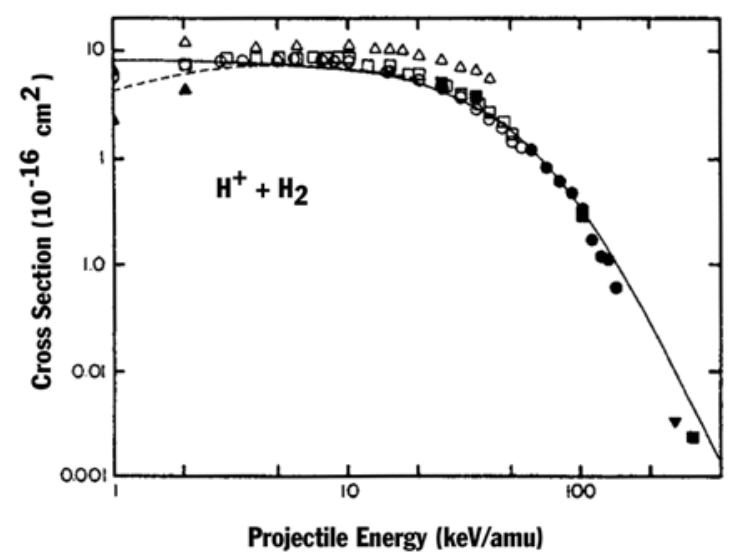

Fig. 3. Single electron capture cross sections for $\mathrm{H}^{+}+\mathrm{H}_{2}$ collisions. The experimental data are from the compilation [10]. Solid line is from the alignment-dependent model [9], and the dashed lines at lower energies are from the MO model [3].

In another example the total charge transfer cross sections for $\mathrm{He}^{2+}+\mathrm{H}_{2}$ collisions in the 10-400 keV/amu energy region are shown in Fig. 4. For this system in this energy region, the dominant electron capture channels are to the $1 \mathrm{~s}, 2 \mathrm{~s}$ and $2 \mathrm{p}$ states of $\mathrm{He}^{+}$. These atomic states on the projectile, together with the $\mathrm{H}(1 \mathrm{~s})$ orbital of the model atom in $\mathrm{H}_{2}$, are used in the $\mathrm{AO}$ expansion calculation. The alignment-averaged cross sections calculated are in good agreement with the experimental data $[11,12]$.

In estimating cross sections for ion- $\mathrm{H}_{2}$ collisions at higher energies, it is customary to assume that the cross section is twice the cross section of ion-H collisions at the same collision velocity. In Fig. 5 we show this ratio for $\mathrm{H}^{+}$and $\mathrm{He}^{2+}$ projectiles in the 10-500 $\mathrm{keV} / \mathrm{amu}$ energy region. Clearly the ratio is not two. A factor of two is obtained if one assumes the phase in Eq. (7) is completely random such that the scattering probabilities from each atomic center can be added incoherently. The phase factor included in Eq.(7) accounts for the coherence, and shows that the phase depends on the collision velocity $\mathrm{v}$, the equilibrium distance of the molecule and the alignment of the molecule with respect to the ion-beam direction. Accounting for such phases can also explain the difference in the so-called $3^{\circ}$ oscillations in $\mathrm{H}^{+}+\mathrm{H}$ and $\mathrm{H}^{+}+\mathrm{H}_{2}$ collisions. In their pioneering experiment in 1962, Lockwood and Everhart[13] measured the charge transfer probability at a scattering angle of $3^{\circ}$ by changing the collision velocity. For $\mathrm{H}^{+}+\mathrm{H}$ collisions they observe that the probability oscillates between 0.1 and 0.9 for 


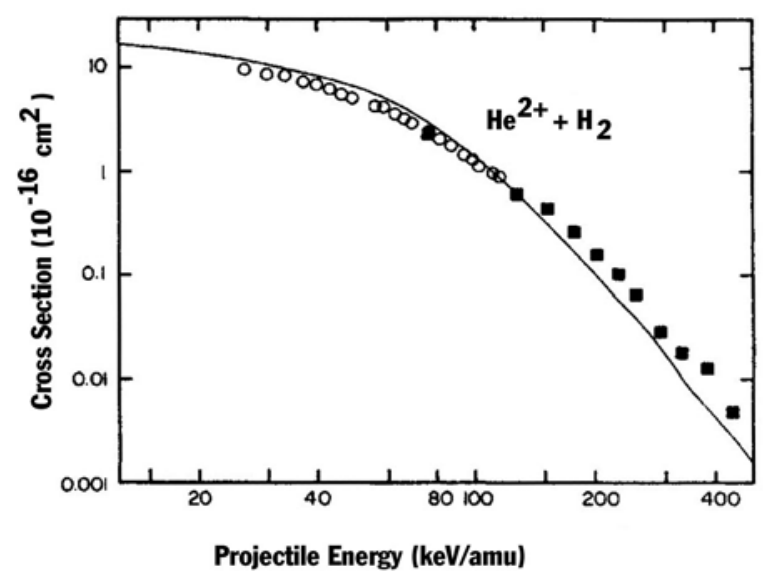

Fig. 4. Single electron capture cross sections in $\mathrm{He}^{2+}+\mathrm{H}_{2}$ collisions. The solid line is from the alignment-dependent atomic model [9] and experiments are from [11] and [12].

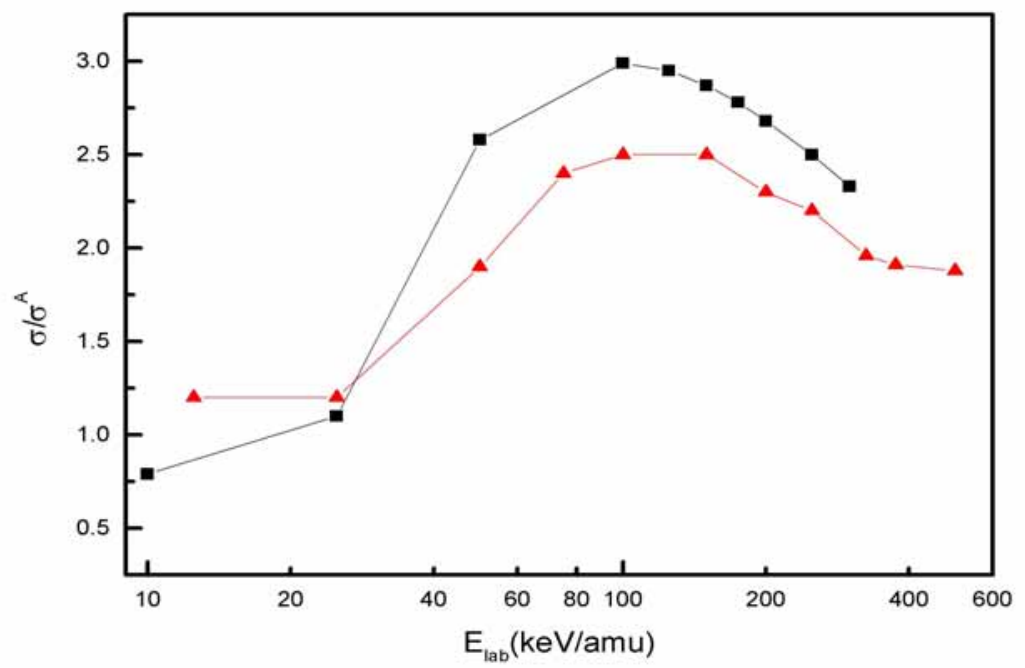

Fig. 5. Ratio of electron capture cross sections from $\mathrm{H}_{2}$ to that from $\mathrm{H}$. The projectiles are $\mathrm{H}^{+}$ (squares) and $\mathrm{He}^{2+}$ (triangles). Data from [9].

proton energy between 1 and $50 \mathrm{keV}$. For $\mathrm{H}^{+}+\mathrm{H}_{2}$, the probability also oscillates with nearly the same phase, but between 0.2 and 0.5 . By averaging over the alignment angles of the $\mathrm{H}_{2}$ molecule, the $3^{\circ}$ oscillation in $\mathrm{H}^{+}+\mathrm{H}_{2}$ data finally was interpreted by Shingal and Lin[14].

\section{$\mathrm{He}^{2+}$ and $\mathrm{Ar}^{2+}$ collisions with $\mathrm{H}_{2}^{+}$}

To examine the dependence of electron capture probabilities on the alignment of molecules, it is crucial to perform an experiment where the data can be interpreted without ambiguity. With $\mathrm{H}_{2}{ }^{+}$as targets, such goal can be achieved. For ions with energies in the order of $\mathrm{keV}$ or higher, the $\mathrm{H}_{2}{ }^{+}$can be treated as frozen in space during the collision, with the electron in the $1 \mathrm{~s} \sigma$ molecular orbital and the two protons at their equilibrium separation of 2 a.u.. Right after the electron is transfered to the projec- 
tile, the two protons find themselves without the screening from the electron. The Coulomb repulsion between the two protons gives each proton a momentum in a direction characterizing the alignment of the $\mathrm{H}_{2}^{+}$molecule.

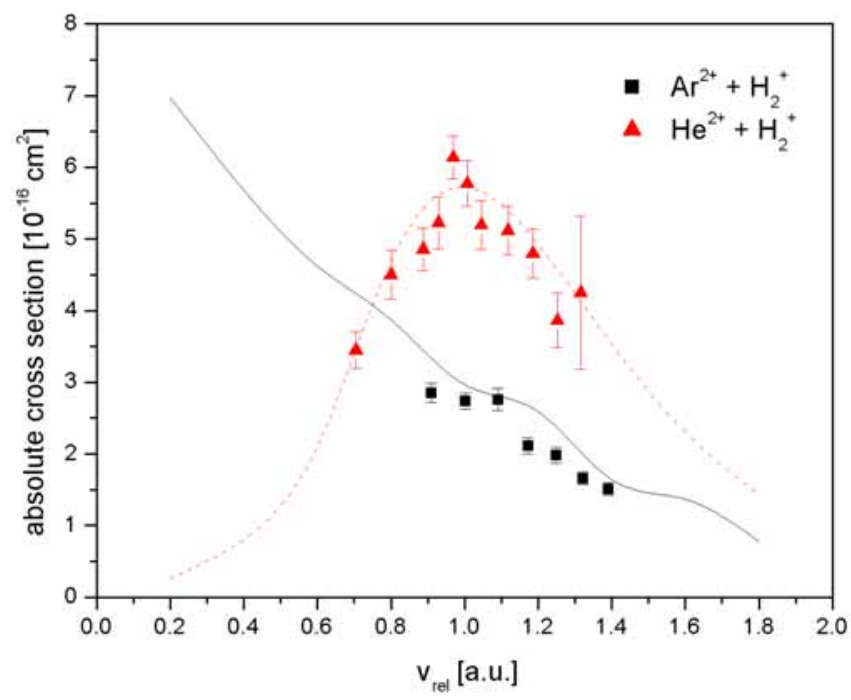

Fig. 6. Absolute total cross sections for electron capture from $\mathrm{H}_{2}^{+}$by $\mathrm{Ar}^{2+}$ and $\mathrm{He}^{2+}$. Symbols: experimental data, lines: alignment-dependent model. From [8].

Before the alignment effect can be explored, it is important to check the alignment averaged total charge transfer cross sections from the $\mathrm{H}_{2}{ }^{+}$target. In Fig. 6 the total charge transfer cross sections for $\mathrm{He}^{2+}+\mathrm{H}_{2}{ }^{+}$and $\mathrm{Ar}^{2+}+\mathrm{H}_{2}{ }^{+}$collisions are shown [8]. The experimental data were taken at Giessen. In the theoretical calculation using the atomic model, the ground state electronic wavefuction is written as the linear combination of hydrogenic 1 s orbitals on each center with an effective charge of $Z^{*}=1.25$. When calculating the ground state energy through the LCAO (linear combination of atomic orbitals) method with a variable effective charge $Z^{*}$, this effective charge minimizes the ground state energy at the equilibrium distance, $\mathrm{R}_{0}=2$ a.u. . The ground state energy at this separation is 1.13 a.u. This is by about half an atomic unit larger than the ionization potential of $\mathrm{H}_{2}^{+}(0.6$ a.u.). This increase is caused by the proximity of the second proton at 2 a.u.

With these parameters the total charge transfer cross sections are calculated using the atomic model described in Section II and the results are shown to be in good agreement with the measurements

Experiments to measure alignment dependent electron capture cross sections for $\mathrm{Ar}^{2+}$ projectiles are being carried out at Kansas State University. The data will be used to compare with the predictions from the atomic model of Shingal and Lin. It is also desirable that full molecular-orbital calculations be carried out as well in the future to test the validity of the atomic model and to check against the experimental data.

\section{Summary}

In this paper we address the alignment-dependent atomic model to obtain electron capture cross sections for ion-molecule collisions. While it is desirable to perform ab initio close-coupling calculations for ion-molecule collisions, this simple model offers the chance to understand the origin of the align- 
ment dependence. The calculation is relatively simple to offer an opportunity to explain or predict ex-

perimental measurements. The recent application of this model to collisions between ions with $\mathrm{H}_{2}^{+}$in a crossed-beam experiment indicates that the model can explain the measured alignment-averaged total charge transfer cross sections. With the pending measurements of the alignment-dependent electron capture cross sections, the prediction from this simple model can be tested at a more fundamental level soon.

\section{Acknowledgement}

This work was supported in part by Chemical Sciences, Geosciences and Biosciences Division, Office of Basic Energy Sciences, Office of Science, U. S. Department of Energy.

\section{References and Notes}

1. Fritsch, W; Lin, C. D. Phys. Rept. 1991, 201, 1.

2. Kimura and Lane, Adv. At. Mol. Opt. Phys. 1990, 26, 79.

3. Kimura, M. Phys. Rev. 1985, A32, 802.

4. Errea, L. F.;Gorfinkiel, J. D.;Macias, A.;Méndez, L; Riera, A. J. Phys. 1999, B32, 1705.

5. Chen, S; Cocke, C. L.; Kamber, E.Y.; Hsu, C. C.; Varghese, S. L. Phys. Rev. 1990, A42, 214.

6. Wang, Y. D.; McGuire, J. H. Phys. Rev. 1991; A44, 367.

7. Rinn, K; Melchert, F; Salzborn, E. J. Phys. 1985, B18, 3783.

8. Brauning, H; Rieser, I.; Diehl, A.; Theiss, A.; Sidky, E.; Cocke, C. L.; Salzborn, E. J. Phys. 2001, B34, L321.

9. Shingal R.; Lin, C. D. Phys. Rev. 1989, A40, 1302.

10. Tawara, H; Kato, T; Nakao, Y. At. Data Nucl. Data Tables, 1985, 32, 235.

11. Hvelplund, P; Anderson, A. Phys. Scripta 1982, 26, 375.

12. Shah, M. B.; Gilbody, H. B. J. Phys. 1981, B14, 2361.

13. Lockwood, G. J.; Everhart, E. Phys. Rev. 1962, 125, 567.

14. Shingal, R.; Lin, C. D. J. Phys. 1989, B22, L659.

(C) 2002 by MDPI (http://www.mdpi.org), Basel, Switzerland. 